\title{
Risky behaviour and non-vaccination
}

\author{
Florence Neymotin ${ }^{1,2}$ (D)
}

Accepted: 3 March 2021 / Published online: 15 April 2021

(C) The Author(s), under exclusive licence to Springer Science+Business Media, LLC, part of Springer Nature 2021

\begin{abstract}
Widespread vaccination acceptance is of critical import to society dealing with the continuing aftermath of the COVID-19 pandemic. The risky behaviours that predict whether individuals vaccinate for seasonal influenza can help policymakers fashion plans to improve vaccination rates and more reliably establish herd immunity. To this end, Canadian Community Health Survey (CCHS) data were employed to determine how an individual's choice to engage in various risky behaviours relates with the likelihood that the same individual gets the seasonal influenza vaccine. Controls were included for demographic, geographic, and health insurance factors. In addition to controlling for these factors, regressions were further stratified based upon gender, the presence of children in the home, and age. I found that excess sun exposure, poor oral hygiene, smoking, and unhealthy diet choices correlated with reduced vaccination probability_-both over the subsequent year and for that individual's lifetime. These results have important implications for properly targeting individuals for widespread vaccinations.
\end{abstract}

Keywords Risk-taking $\cdot$ Herd immunity $\cdot$ Influenza $\cdot$ Vaccination

JEL Code I10 $\cdot$ I1 8

\section{Introduction and background}

On March 11, 2020, the World Health Organization declared COVID-19, a potentially life-threatening respiratory disease caused by a novel coronavirus, to be a worldwide pandemic. While the responses of governments around the globe, which included mandated social distancing, quarantines, and the closing of borders, may

Florence Neymotin

fneymotin@nova.edu

1 Huizenga College of Business, Nova Southeastern University, 3301 College Ave., Fort Lauderdale, FL 33314, USA

2 Sprott School of Business, Carleton University, Ottawa, Canada 
have seemed unthinkable just months before, many epidemiologists and those charged with disaster preparedness had long anticipated the grim possibility of a novel contagion against which the population had no previous immunity. The present situation highlights the need for a robust policy to help maximize vaccine adoption for all viruses, and particularly those looming strains of seasonal influenza that still cause hundreds of thousands of deaths annually.

The present analysis focuses on a variety of risky behaviours and how they correlate with the tendency to receive the seasonal influenza vaccination. Due to the particularly rich and recent dataset on these issues in Canada, I have focused the analysis there, with implications easily transferrable to vaccination more generally.

\subsection{Background}

In an interesting recent paper by Walter, Ophir and Jamieson (2020) the authors showed that Twitter bots programmed to exhibit pro- or anti-Trump feelings displayed clear polarization on the issue of vaccinations. Similarly, older individuals appear more likely to get the influenza vaccine when it is covered in the news (Yoo et al., 2010), and "framing" vaccinations in a certain way can affect whether an individual chooses to get it, such as the one for HPV (LeFevre et al., 2016; Nan, Zie, \& Madden, 2012). On the other hand, there are limits to the persuasive power of new information, as in a study of college students who did not change their minds from their initial vaccination stances (Bronchetti, Huffman, \& Magenheim, 2015). However, they did seem to respond to financial incentives, and, similarly, physicians have been observed to vaccinate more patients after either they or the patients were given financial renumeration (Ibuka \& Bessa, 2016; Kontopantelis et al., 2012). It is also important that the information presented be as accurate as possible (Robra \& Felder, 2020). A recent study (Murphy et al., 2021) on the complex psychological processes that undergird vaccine hesitancy show that many factors are implicated, including perceived locus of control, belief system, and trust of authority figures.

While causal relationships are complicated and cannot be inferred from this data, the present analysis makes some initial inroads in answering the question of whether individuals who engage in risky behaviours also tend to get vaccinated less often. ${ }^{1}$ In the context of the preceding background information, the purpose of this study can be seen as identifying relevant populations for informational targeting to create positive frames and ultimately establishing herd immunity.

\footnotetext{
1 As an aside, notice that some of these risky behaviors can be considered from a lack of control perspective in behavioral economics (Burnham and Phelan, 2020).
} 


\section{Materials and methods}

\subsection{Data}

Data for this study come from the 2015-2016 self-reported Canadian Community Health Survey (CCHS). The CCHS is a cross-sectional survey that collects information related to health status, healthcare utilization, and health determinants for the Canadian population. The CCHS covers individuals aged 12 and older who are living within the 10 provinces or the 3 territories. It relies upon a large sample of respondents and is designed to provide reliable health estimates at the region level every 2 years. The survey began collecting data in 2001, and was repeated every 2 years until 2005. Starting in 2007, data for the CCHS were collected annually instead of every 2 years. While a sample of approximately 130,000 respondents were interviewed during the reference periods of 2001, 2003 and 2005 , the sample size was changed to 65,000 respondents each year starting in 2007.

In terms of access, all researchers with a university affiliation at the Data Liberation Initiative in Canada can obtain the data. The dataset was chosen due to its detailed information on the demographic characteristics of the individuals filling out the survey, risky individual behaviours, and specific choices regarding influenza vaccination. There are few comparable recent surveys available anywhere in the world. Finally, with respect to the concern that respondents do not always honestly report their vaccination status, recent work indicates that individual retrospective answers on this question are fairly accurate (Smolinski et al., 2015).

In terms of demographics, individuals were asked their age, income, marital status, gender, whether children of school-age are present in the home, province of residence, highest education achieved, as well as other factors not employed here.

Respondents were also asked about their dental insurance situation, since it is not covered by the Canada Health Act. Since essentially everyone residing in Canada is covered by a health insurance plan provided by their province, I cannot employ differentiation as in Courtemanche et al (2018). To examine the effect of out-of-pocket healthcare costs on risk taking. Instead, I use dental insurance as an additional indicator of income security-while also using income itself, since dental insurance is often paid for by employers, or can be purchased independently by individuals if not offered at their workplace.

To determine risky behaviours, the following definitions were employed:

- Hazardous Driving any of (a) had and drove a motorcycle, (b) texted all the time, or (c) drove more than three times in the last twelve months when they were either tired, had too much to drink, or used illicit substances.

- Unprotected Sports rarely or never wore the necessary safety equipment for any of bicycling, roller blading, skiing, snowboarding, skateboarding, ice hockey, or inline skating.

- Excessive Sun Exposure any of (a) had a sunburn in the last twelve months, (b) spent more than $2 \mathrm{~h}$ regularly in the sun during peak (10 a.m. to 4 p.m.) in 


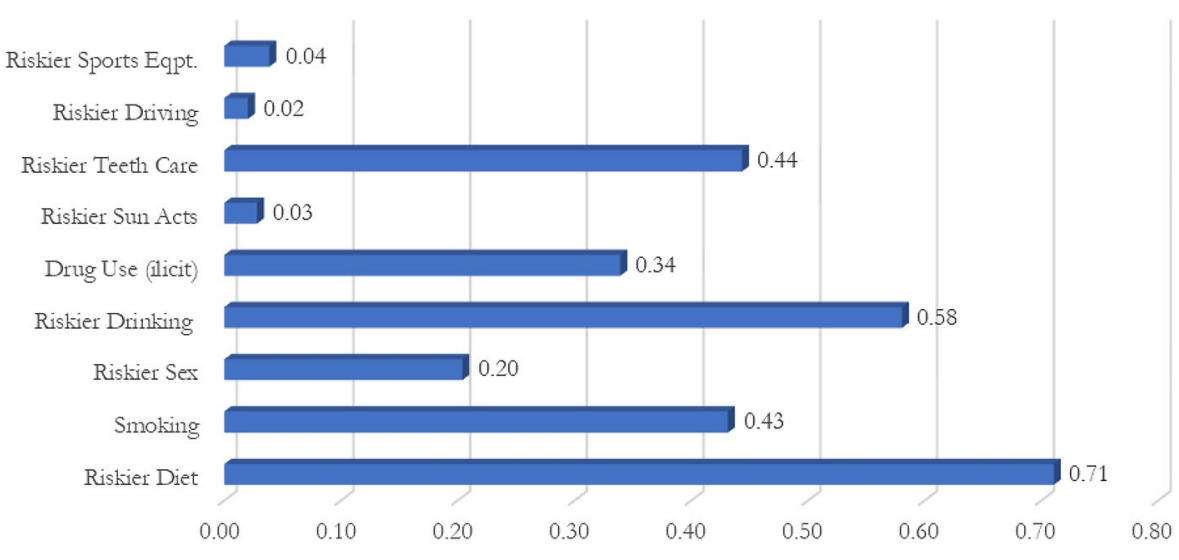

Fig. 1 Fraction engaging in risky behaviors

the summer, (c) rarely or never sought shade in the sun in summer, wore sunglasses, or used sunscreen, (d) used a tanning bed this year, or (e) generally didn't protect themselves from the sun.

- Poor Dental hygiene did not brush and floss at least once a day.

- Alcohol drank regularly or had more than 10 drinks a week on a regular basis.

- Illicit drug use engaged in any illegal drug use at any point during their lifetime (marijuana excepted).

- Smoking considered themselves daily or occasional smokers or used any smoking-related items including cigars, electronic cigarettes, pipes, chewing tobacco, or tobacco water pipes.

- Risky Sex did not use protection of any form when not in a monogamous relationship or expecting a child (emergency contraceptives excluded).

- Poor Diet did not plan meals based on nutritional guidelines or avoided unhealthy foods.

Figure 1 displays the specific fraction of individuals who engaged in each of the risky behaviours listed above. It is apparent that few individuals rigorously follow health guidelines, since many individuals drink, smoke, and/or do not properly care for their teeth. On the other hand, very few individuals display excessive sun exposure, risky driving, or play sports without protective equipment. Nevertheless, the fractions are reasonable and not so extreme so that they cannot yield useful results in the regression analysis that follows.

Individuals were asked whether they had "ever" received a flu shot, and, those who answered in the affirmative were further asked to date their most recent flu shot as having occurred within the last year (now), up to 2 years ago, or greater than 2 years prior. As seen in Fig. 2, there was some variation by province in the percentage of individuals who had ever received a flu shot, with Quebec (50\%) lagging behind Ontario by 17 percentage points, and Prince Edward Island by 24. This clear variation by location confirmed the need to control for province of residence in the later portion of this analysis. 


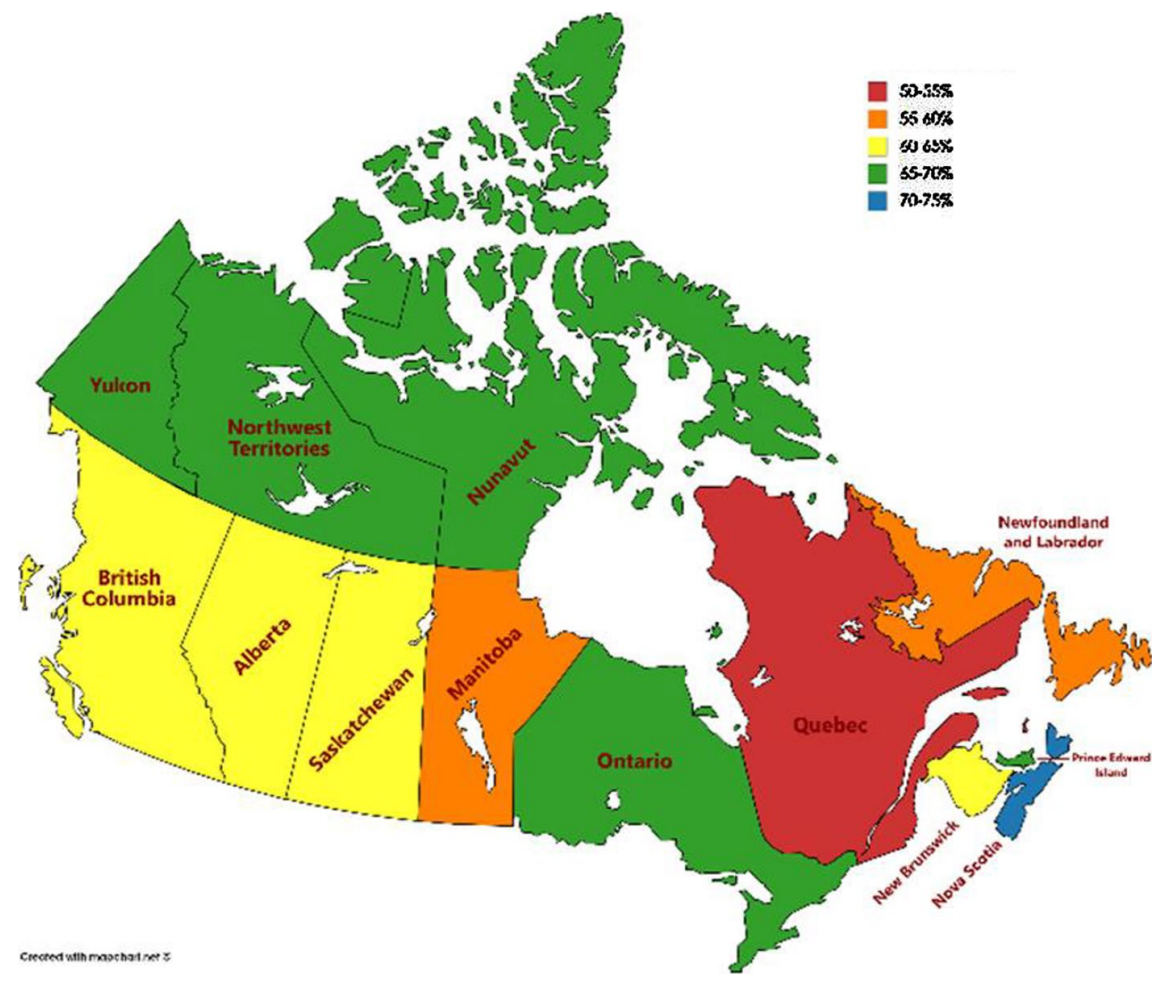

Fig. $2 \%$ Ever receiving flu vaccine (CCHS)

In terms of the control variables, Table 1 displays the means of variables employed in the analysis. With an average age of 49 , and with $54 \%$ Female, the sample is approximately what one would expect for an analysis of this form. It should be noted that a somewhat larger fraction of the sample than typical has completed postsecondary education (56\%), over half are married or cohabiting, and only $11 \%$ have dental insurance. ${ }^{2}$ This likely reflects differences for Canada in particular.

\subsection{Structure}

The main research question this work addresses is whether engaging in riskier behaviours was positively or negatively correlated with the chance of obtaining the vaccination for seasonal influenza. I considered both recent influenza vaccinations and whether an individual had "ever received a flu shot".

In addition to examining the choice of risky behaviours in detail, I controlled for each of the demographic factors listed previously (income, education, age and age squared, marital status, presence of children, and gender) as well as province and access to dental insurance. Regression analyses were performed using a linearized

\footnotetext{
2 The sample size (N) ranges from 106,002 to 109,659 depending on responses for that particular question-regressions are drawn from a slightly smaller sample.
} 
Ordinary Least Squares (OLS) regression to predict the likelihood of influenza vaccinations based on the presence or absence-or the levels when the variable was continuous - of all of the previous listed variables. Marginal Probit regressions were also performed in robustness analyses and yielded virtually identical patterns.

Table 1 Control variable means

\begin{tabular}{ll}
\hline Dental Insurance & $11 \%$ \\
\hline Income $<\$ 20 \mathrm{~K}$ & $10 \%$ \\
$\$ 20 \mathrm{~K}<$ Income $<\$ 40 \mathrm{~K}$ & $19 \%$ \\
$\$ 40 \mathrm{~K}<$ Income $<\$ 60 \mathrm{~K}$ & $17 \%$ \\
$\$ 60 \mathrm{~K}<$ Income $<\$ 80 \mathrm{~K}$ & $14 \%$ \\
$\$ 80 \mathrm{~K}<$ Income & $41 \%$ \\
Female & $54 \%$ \\
Has Kids & $20 \%$ \\
Married or Cohabiting & $51 \%$ \\
Divorced/Widowed/Separated & $20 \%$ \\
Single (marital status) & $29 \%$ \\
Age & 48.95 \\
Educ: Didn't Complete Secondary School & $23 \%$ \\
Educ: Didn't Complete Post-Secondary School & $21 \%$ \\
Educ: Completed Secondary School & $56 \%$ \\
\hline
\end{tabular}

\section{Results}

\subsection{Regressions}

After controlling for demographics and province, Tables 2 and 3 show how risky behaviours predict an individual's tendency to either receive a vaccination for influenza in the previous year or ever in their lifetime. Regressions are stratified by age (all ages versus over 27 years old), gender, and whether an individual has children present in the home, as well as controlling for those variables when possible-i.e. when not stratifying (coefficients results available upon request). ${ }^{3}$

Table 2 uses OLS regressions and reveals that several risky behaviours have a statistically significant (five percent or one percent) relationship with having received a flu shot in the past year. The size of the sample is relatively large, with 104,594

\footnotetext{
3 Due to a concern that multicollinearity might bias the results, a full correlation matrix was constructed including all of the different risky variables. Of the 36 entries, only 6 correlations exceeded 0.2 (magnitude), and only 2 of these 6 exceeded 0.3 . In particular, there was a -0.3693 correlation between risky sex and using drugs, and a -0.3587 correlation between risky sun exposure and riskier food choices. I considered this within reasonable bounds to continue to include the variables jointly in the analysis.
} 
Table 2 Risky behaviors and having a flu shot this year

\begin{tabular}{|c|c|c|c|c|c|c|}
\hline AGE & ALL & $>27$ & $>27$ & $>27$ & $>27$ & $>27$ \\
\hline GENDER & ALL & ALL & MALE & FEMALE & ALL & ALL \\
\hline \multirow[t]{2}{*}{ FAMILIES } & ALL & ALL & ALL & ALL & NO KIDS & HAVE KIDS \\
\hline & {$[1]$} & {$[2]$} & {$[3]$} & [4] & {$[5]$} & {$[6]$} \\
\hline \multirow[t]{2}{*}{ Riskier Diet } & -0.054 & -0.058 & -0.065 & -0.058 & -0.066 & -0.038 \\
\hline & {$[11.96]^{* *}$} & {$[11.64]^{* *}$} & {$[8.83]^{* *}$} & {$[8.36]^{* *}$} & {$[11.60]^{* *}$} & {$[3.58]^{* *}$} \\
\hline \multirow[t]{2}{*}{ Smoking } & -0.057 & -0.06 & -0.068 & -0.049 & -0.065 & -0.036 \\
\hline & {$[15.54]^{* *}$} & {$[15.06]^{* *}$} & {$[12.59]^{* *}$} & {$[8.57]^{* *}$} & {$[14.57]^{* *}$} & {$[4.05]^{* *}$} \\
\hline \multirow[t]{2}{*}{ Riskier Sex } & 0.022 & 0.022 & 0.028 & 0.015 & 0.024 & -0.001 \\
\hline & {$[5.03]^{* *}$} & {$[4.55] * *$} & {$[4.11]^{* *}$} & {$[2.12]^{*}$} & {$[4.70]^{* *}$} & {$[0.10]$} \\
\hline \multirow[t]{2}{*}{ Riskier Drinking } & -0.001 & 0 & -0.008 & 0.006 & 0.004 & -0.018 \\
\hline & {$[0.36]$} & {$[0.13]$} & {$[1.57]$} & {$[1.43]$} & [1.17] & {$[2.39]^{*}$} \\
\hline \multirow[t]{2}{*}{ Drug Use (illicit) } & -0.007 & -0.006 & -0.002 & -0.009 & -0.005 & -0.011 \\
\hline & {$[2.23]^{*}$} & [1.58] & {$[0.43]$} & {$[1.78]$} & {$[1.33]$} & {$[1.54]$} \\
\hline \multirow[t]{2}{*}{ Riskier Sun Acts } & -0.028 & -0.028 & -0.036 & -0.032 & -0.026 & -0.038 \\
\hline & {$[6.50]^{* *}$} & {$[6.21]^{* *}$} & {$[4.98]^{* *}$} & {$[5.27]^{* *}$} & {$[5.21]^{* *}$} & {$[3.07]^{* *}$} \\
\hline \multirow[t]{2}{*}{ Riskier Teeth Care } & -0.046 & -0.042 & -0.049 & -0.024 & -0.035 & -0.063 \\
\hline & {$[5.11]^{* *}$} & {$[4.13]^{* *}$} & {$[3.80]^{* *}$} & {$[1.48]$} & {$[3.09]^{* *}$} & {$[2.98]^{* *}$} \\
\hline \multirow[t]{2}{*}{ Riskier Driving } & -0.031 & -0.018 & -0.022 & 0.012 & -0.018 & -0.022 \\
\hline & {$[2.93]^{* *}$} & [1.59] & {$[1.54]$} & {$[0.60]$} & {$[1.36]$} & {$[1.03]$} \\
\hline \multirow[t]{2}{*}{ Riskier Sports Eqpt } & -0.022 & -0.034 & -0.019 & -0.045 & -0.033 & -0.056 \\
\hline & {$[2.64]^{* *}$} & {$[3.56]^{* *}$} & {$[1.49]$} & {$[2.98]^{* *}$} & {$[2.76]^{* *}$} & {$[3.26]^{* *}$} \\
\hline \multirow[t]{2}{*}{ Constant } & 0.389 & 0.392 & 0.445 & 0.434 & 0.351 & 0.398 \\
\hline & {$[29.87]^{* *}$} & {$[19.04]^{* *}$} & {$[14.90]^{* *}$} & {$[15.31]^{* *}$} & {$[15.31]^{* *}$} & {$[6.83]^{* *}$} \\
\hline $\mathrm{R}^{2}$ & 0.15 & 0.15 & 0.17 & 0.12 & 0.15 & 0.05 \\
\hline $\mathrm{N}$ & 104,594 & 89,550 & 40,475 & 49,075 & 72,683 & 16,867 \\
\hline
\end{tabular}

Coefficients are displayed with t-statistics below in brackets. Additional binary controls were included for each of the provinces, income groupings, whether the individual had dental insurance or not, marital status in three categories, education in three categories, and a continuous variable for age and its square

*Significant at the $5 \%$ level and **significant at the $1 \%$ level

observations in the first group. Having excessive sun exposure, poor oral hygiene at home, playing sports without protection, making bad diet choices, risky sex, and smoking all make it more likely that the individual did not receive the flu shot in the previous year. Risky driving and illicit drug use each have a relationship with flu vaccines only when considering younger, but not older individuals.

Table 3 repeats the analysis from Table 2, but with the outcome of having ever received a flu shot rather than, just in the past year. The patterns are similar to the previous section, with, once again, subpar oral hygiene, smoking, and bad diet choices predicting a smaller chance for influenza vaccinations.

On the other hand, the safe use of sports equipment no longer appears significant. In the longer term, it is also true that riskier sex behaviours seem to predict less vaccinations rather than more, and, interestingly, illicit drug use consistently makes it 
Table 3 Risky behaviors and "Ever" having a flu shot

\begin{tabular}{|c|c|c|c|c|c|c|}
\hline AGE & ALL & $>27$ & $>27$ & $>27$ & $>27$ & $>27$ \\
\hline GENDER & ALL & ALL & MALE & FEMALE & ALL & ALL \\
\hline \multirow[t]{2}{*}{ FAMILIES } & ALL & ALL & ALL & ALL & NO KIDS & HAVE KIDS \\
\hline & [1] & {$[2]$} & [3] & [4] & {$[5]$} & [6] \\
\hline \multirow[t]{2}{*}{ Riskier Diet } & -0.072 & -0.074 & -0.076 & -0.077 & -0.077 & -0.067 \\
\hline & {$[15.31]^{* *}$} & {$[14.42]^{* *}$} & {$[9.64]^{* *}$} & {$[11.17]^{* *}$} & {$[13.45]^{* *}$} & {$[5.65]^{* *}$} \\
\hline \multirow[t]{2}{*}{ Smoking } & -0.048 & -0.051 & -0.07 & -0.032 & -0.056 & -0.03 \\
\hline & {$[12.30]^{* *}$} & {$[12.56]^{* *}$} & {$[12.03]^{* *}$} & {$[5.57]^{* *}$} & {$[12.37]^{* *}$} & {$[3.07] * *$} \\
\hline \multirow[t]{2}{*}{ Riskier Sex } & -0.018 & -0.012 & -0.01 & -0.015 & -0.009 & -0.045 \\
\hline & {$[4.01]^{* *}$} & {$[2.36]^{*}$} & [1.29] & {$[2.25]^{*}$} & [1.66] & {$[2.80]^{* *}$} \\
\hline \multirow[t]{2}{*}{ Riskier Drinking } & 0.008 & 0.008 & -0.004 & 0.016 & 0.007 & 0.006 \\
\hline & {$[2.44]^{*}$} & {$[2.25]^{*}$} & {$[0.75]$} & {$[3.53]^{* *}$} & [1.83] & {$[0.70]$} \\
\hline \multirow[t]{2}{*}{ Drug Use (illicit) } & 0.019 & 0.022 & 0.022 & 0.023 & 0.019 & 0.029 \\
\hline & {$[5.58]^{* *}$} & {$[5.99] * *$} & {$[4.17]^{* *}$} & {$[4.47]^{* *}$} & {$[4.57]^{* *}$} & {$[3.59]^{* *}$} \\
\hline \multirow[t]{2}{*}{ Riskier Sun Acts } & -0.015 & -0.014 & -0.013 & -0.019 & -0.014 & -0.002 \\
\hline & {$[3.24]^{* *}$} & {$[2.92]^{* *}$} & {$[1.66]$} & {$[3.25]^{* *}$} & {$[2.71]^{* *}$} & {$[0.13]$} \\
\hline \multirow[t]{2}{*}{ Riskier Teeth Care } & -0.074 & -0.065 & -0.062 & -0.058 & -0.057 & -0.089 \\
\hline & {$[7.72]^{* *}$} & {$[6.27]^{* *}$} & {$[4.47]^{* *}$} & {$[3.60]^{* *}$} & {$[4.96]^{* *}$} & {$[3.80]^{* *}$} \\
\hline \multirow[t]{2}{*}{ Riskier Driving } & -0.026 & -0.015 & -0.005 & -0.009 & -0.016 & -0.017 \\
\hline & {$[2.33]^{*}$} & {$[1.28]$} & {$[0.36]$} & {$[0.44]$} & {$[1.18]$} & {$[0.70]$} \\
\hline \multirow[t]{2}{*}{ Riskier Sports Eqpt } & 0 & -0.009 & 0.009 & -0.025 & -0.012 & -0.022 \\
\hline & {$[0.02]$} & {$[0.88]$} & {$[0.69]$} & {$[1.65]$} & {$[1.03]$} & [1.17] \\
\hline \multirow[t]{2}{*}{ Constant } & 0.774 & 0.72 & 0.844 & 0.676 & 0.718 & 0.588 \\
\hline & {$[56.48]^{* *}$} & {$[34.00]^{* *}$} & {$[26.21]^{* *}$} & {$[24.07]^{* *}$} & {$[30.91]^{* *}$} & {$[9.13] * *$} \\
\hline $\mathrm{R}^{2}$ & 0.07 & 0.08 & 0.09 & 0.07 & 0.09 & 0.05 \\
\hline $\mathrm{N}$ & 104,594 & 89,550 & 40,475 & 49,075 & 72,683 & 16,867 \\
\hline
\end{tabular}

Coefficients are displayed with t-statistics below in brackets. Additional binary controls were included for each of the provinces, income groupings, whether the individual had dental insurance or not, marital status in three categories, education in three categories, and a continuous variable for age and its square

*Significant at the $5 \%$ level and **significant at the $1 \%$ level

more likely that an individual will ever have a vaccination for influenza. This may be because those individuals are more likely to be engaged by the healthcare system due to issues stemming from risky sexual behaviour and may subsequently be asked about vaccinations during their interactions with providers. ${ }^{4}$

\footnotetext{
4 In an additional test, Quebec was separately compared to Ontario for both the outcome of "ever having a flu shot" as well as "having a flu shot this year." In all cases, smoking and poor diet choices negatively affected the likelihood of vaccination. Excessive sun exposure negatively affected the likelihood of vaccination in both provinces on a yearly, but not on a lifetime, basis-similarly to the full regression results.
} 


\section{Discussion}

This work employed CCHS data to identify the risky actions that help predict the likelihood that an individual will choose to partake in the seasonal influenza vaccine. Excessive sun exposure, poor oral hygiene, smoking, and unhealthy diet choices made vaccinations less likely in both the previous year and over the course of a lifetime. These patterns were confirmed in robustness analyses that used different functional forms. The findings imply clear relationships between an individual's choice to engage in risky behaviours and their decision to forego vaccination.

The present results also concord with others in the literature. For example, Buchan and Kwong (2016) employed the CCHS and found that vaccination varied by province, and that it was less likely among older individuals (Hanoch, Rolison, \& Freund, 2016) — with the present analysis having age show a positive coefficient and a negative quadratic.

The findings of the present analysis also agree with those of Dick and Nordstrom (2016), who used the CCHS to show that smoking was negatively correlated with the likelihood of vaccination. Those authors also found that marital status and having young children affected the prevalence of vaccinations, as seen here. However, their analysis imposed a Random Forest Machine Learning Model and used all the data without any a priori justification for the variables. This approach is sometimes useful, but it is susceptible to multicollinearity problems and can yield results that lack interpretability.

Regarding drinking, French, Ettner and Popovici (2010) were able to causally demonstrate that alcohol affected risk-taking, which included the decision to receive a flu vaccine (negatively). This stands in contrast to the present work, in which individuals who drank more or used illicit drugs were more likely to receive the vaccination. I would presume the difference between these results may lie in my definition of alcohol risk-taking and perhaps in why people choose to forego the vaccine.

\subsection{Limitations}

This analysis is intended to discover important correlations between risky choices and the act of obtaining a vaccine, with the goal of targeting individuals who may benefit from additional information. This information will, hopefully, increase the rate of vaccinations. The low $\mathrm{R}^{2}$ in this study's regressions, are not entirely surprising, as many other factors determine vaccination rates, and a lower $\mathrm{R}^{2}$ is common for a study of this form. Some omitted factors may turn out to be important when compared with the characteristics included in the analysis. In fact, while I have done my best to include correlations and stratifications as evidence, the

Footnote 4 (continued)

While the overall pattern of results were the same, there were some expected variations in magnitude between the provinces, likely due at least in part to their varying vaccination policies. 
possibility remains that additional factors are just as, or perhaps more, important in predicting vaccination rates. But given the inherent limitations in predicting human behaviour, especially regarding complex, multifaceted decisions such as the one considered here, the findings of this work may very well be of use to policy-makers. This is particularly true given the difficult, but highly important task of safeguarding public health through vaccination.

Furthermore, the data in this study does not allow me to distinguish between vaccine refusal and vaccine non-availability. While some questions regarding insurance availability, and income and demographics were employed either in the main study or in initial regressions, one could argue that there is selection into the insurance groups so that employing these characteristics will still allow bias to remain. For this reason, I cannot definitively determine whether the correlational relationships are entirely a result of free choice or outside constraints.

\section{Conclusions}

While it is often difficult to assess the complex psychological, sociological, and demographic factors (Travers, Dick, \& Stone, 2018) associated with healthcare choices, and in particular, the choice to get vaccinated, the results of this work help shine a light on some important points of engagement policymakers have with individuals who have not vaccinated in the past. For example, if the problem is that individuals who are more risk-tolerant are also more likely to refuse the seasonal influenza vaccination, which is consistent with my findings, targeted campaigns for this group may tip the balance in favour of getting protection. It is also the case that, although the present study only uses data for seasonal influenza vaccinations, patterns for how individuals view one type of vaccination are likely to spill over to how individuals view other vaccinations (Schulz \& Hartung, 2020). Therefore, the present findings are crucially important in our efforts to achieve mass vaccination for COVID-19.

Additional resources and attention can be allocated to target individuals displaying the risk factors identified in this work in order to increase the efficacy of the present vaccinations. While viral threats will likely always remain, we can try to implement cost-effective public policies to enhance vaccination rates and protect vulnerable populations.

Author contributions Sole-authored work.

Funding The author has no funding to report for this work.

Data availability Data were made available through participation in the university-level Canadian Data Liberation Initiative. Canadian researchers and those who apply can secure this data.

Code availability Code will be provided upon request. 


\section{Declarations}

Conflict of interest The author has no conflicts of interest to disclose.

\section{References}

Bronchetti, E. T., Huffman, D. B., \& Magenheim, E. (2015). Attention, intentions, and follow-through in preventative health behaviour: Field experimental evidence on flu vaccination. Journal of Economic Behavior and Organization, 116, 270-291.

Buchan, S. A., \& Kwong, J. C. (2016). Trends in influenza vaccine coverage and vaccine hesitancy in Canada, 2006/07 to 2013/14: Results from cross-sectional survey data. CMAJ Open, 4(3), E455-E462.

Burnham, T. C., \& Phelan, J. (2020). Ordinaries. Journal of Bioeconomics, 22, 137-154.

Courtemanche, C., Marton, J., Ukert, J., Yelowitz, A., \& Zapata, D. (2018). Effects of the affordable care act on health behaviors after three years. NBER Working Paper No. 24511.

Dick, K., \& Nordstrom, A. (2016). Identifying unvaccinated individuals in Canada: A predictive model. arXiv: 1607.08656

French, M. T., Ettner, S. L., \& Popovici, I. (2010). Heavy drinking and health promotion activities. Social Science and Medicine, 71(1), 134-142.

Hanoch, Y., Rolison, J. J., \& Freund, A. M. (2016). Does medical risk perception and risk-taking change with age? Risk Analysis, 38(5), 917-928.

Ibuka, Y., \& Bessa, S. I. (2016). Out-of-pocket payments and community-wide health outcomes: An examination of influenza vaccination subsidies in Japan. Health Economics, Policy and Law, 11(3), 275-302.

Kontopantelis, E., Doran, T., Gravelle, H., Goudie, R., Siciliani, L., \& Sutton, M. (2012). Family doctor responses to changes in incentives for influenza immunization under the quality and outcomes framework pay-for-performance scheme. Health Services Research, 47(3pt1), 1117-1136.

LeFevre, E., Hens, N., De Smet, F., \& Beutels, P. (2016). The impact of non-financial and financial encouragements on participation in non-school-based human papillomavirus vaccination: A retrospective cohort study. The European Journal of Health Economics, 17, 305-315.

Murphy, J., Vallières, F., Bentall, R. P., Shevlin, M., McBride, O., Hartman, T. K., McKay, R., Bennett, K., Mason, L., Gibson-Miller, J., Levita, L., Martinez, A. P., Stocks, T. V. A., Karatzias, T., \& Hyland, P. (2021). Psychological characteristics associated with COVID-19 vaccine hesitancy and resistance in Ireland and the United Kingdom. Nature Communications, 12(1), 1-15.

Nan, Z., Zie, B., \& Madden, K. (2012). Acceptability of the H1N1 vaccine among older adults: The interplay of message framing and perceived vaccine safety and efficacy. Health Communications, 27, 559-568.

Robra, B. P., \& Felder, S. (2020). Homburg's lockdown analysis: Conclusions without data and an appropriate estimation model. The Economists' Voice. https://doi.org/10.1515/ev-2020-0015.

Schulz, P. J., \& Hartung, U. (2020). Unsusceptible to social communication? The fixture of the factors predicting decisions on different vaccinations. Health Communication. https://doi.org/10.1080/10410236.2020. 1771119.

Smolinski, M. S., Crawley, A. W., Baltrusaitis, K., Chunara, R., Olsen, J. M., Wojcik, O., Santillana, M., Nguyen, A., \& Brownstein, J. S. (2015). Flu near you: Crowdsourced symptom reporting spanning 2 influenza seasons. American Journal of Public Health, 105(10), 2124-2130.

Travers, J. L., Dick, A. W., \& Stone, P. W. (2018). Racial/ethnic differences in receipt of influenza and pneumococcal vaccination among long-stay nursing home residents. Health Services Research, 53(4), 2203-2226.

Walter, D., Ophir, Y., \& Jamieson, K. H. (2020). Russian Twitter accounts and the partisan polarization of vaccine discourse, 2015-2017. American Journal of Public Health, 110(5), 718-724.

Yoo, B. K., Holland, M. L., Bhattacharya, J., Phelps, C. E., \& Szilagyi, P. G. (2010). Effects of mass media coverage on timing and annual receipt of influenza vaccination among Medicare elderly. Health Services Research, 45(5pt1), 1287-1309.

Publisher's Note Springer Nature remains neutral with regard to jurisdictional claims in published maps and institutional affiliations. 